\title{
UTILIZAÇÃO DA RESERVA ORGÂNICA E DE NITROGÊNIO DO TOLETE DE PLANTIO (COLMO-SEMENTE) NO DESENVOLVIMENTO DA CANA-PLANTA ${ }^{1}$
}

\author{
A.E.V. CARNEIRO ${ }^{3}$; P.C.O. TRIVELIN ${ }^{4,2}$; R.L. VICTORIA ${ }^{4,2}$ \\ ${ }^{3}$ Universidade Metodista de Piracicaba - UNMEP - C.P. 68, CEP: 13400-901, Piracicaba, SP. \\ 'Centro de Energia Nuclear na Agricultura - CENA/USP - C.P. 96, CEP: 13400-970, Piracicaba, SP.
}

\begin{abstract}
RESUMO: Toletes (colmo-semente) com um no (uma gema) mais metade do entre-no inferior e metade do superior foram obtidos do terço médio de colmos de cana-de-açúcar, marcados com ${ }^{15} \mathrm{~N}$, da variedade NA 56-79. A massa seca, o contendo de mitrogennio e a abundância isotopica de ${ }^{15} \mathrm{~N}$ dos toletes foram estimados tomando-se por base os valores obtidos em amostra de cada colmo. Os toletes foram plantados em vasos com $6 \mathrm{~kg}$ de terra colhida de uma Terra Roxa Estruturada Latossflica (RHODIC KANHAPLUDALF). $O$ experimento foi conduzido em casa-devegetação, em delineamento inteiramente casualizado, com 16 tratamentos correspondentes à colheitas em diferentes tempos apos o plantio. Nas plantas, subdivididas em amostras de parte aérea, rázes e tolete remanescente, foram determinados a massa seca, $\mathbf{N}$-total e abundância de ${ }^{13} \mathrm{~N}$. Dos resultados obtidos verificou-se que a redução da matéria seca do tolete somente foi compensada pela produção de novos tecidos por volta dos 3 meses do plantio. Nesse periodo, a emissão das rázes de fixação e a brotação do perfilho primário dependeu da reserva orgânica do tolete. A reserva de nitrogênio do tolete foi fundamental como fonte do nutriente à cana-planta nos 50-60 dias ap6s - plantio. A degradação de 50\% da reserva orgânica do tolete ocorreu até os 6 meses e possibilitou a liberação de 50\% da reserva de nitrogênio que foi transiocada aos novos tecidos da cana-planta. Pode-se estimar que de 6-12 kg.ha-1 do $\mathrm{N}$-tolete são translocados aos novos tecidos ( $50 \%$ do $\mathrm{N}$-tolete), valores estes que podem representar 5-10\% das necessidades de $\mathbf{N}$ da cultura.
\end{abstract}

Descritores: Translocação de N; ${ }^{15} \mathrm{~N}$; N do tolete (colmo-semente); cana-de-açúcar; cana-planta

\section{UTILIZATION OF THE ORGANIC AND NITROGEN RESERVE OF THE SEEDPIECE BY THE CANE PLANT}

\begin{abstract}
Seedpieces with one node (one bud) plus half of the internodes at each side, were cut from the middle third of cane stalks, variety NA 56-79, grown with ${ }^{15} \mathrm{~N}$. The dry weight, $\mathrm{N}$ content and ${ }^{15} \mathrm{~N}$ abundance of cuttings were estimated from samples of each original stalk. The cuttings were planted in pots filled with $6 \mathrm{~kg}$ of a Dark Red Latosol (RHODIC KANIIAPLUDALF) sample. The experiment was conducted under greenhouse conditions, using a random design with 16 treatments (harvest times) and three replicates. The harvested plants were divided into shoots, roots and remaining seedpiece. The dry weight, $N$ content and ${ }^{15} \mathrm{~N}$ abundance were determined in each sample. The results showed that the reduction of the dry weight of the seedpiece was compensated by new matter yield (shoot and root) only after three months of growth. During this period the emergence of fixing roots and of the primary shoot depended solely on the seedpiece reservoir. The later is also essential as a nitrogen source for the cane plant during 50-60 days after planting. After 6 months the dry matter of the original cuttings is reduced by $50 \%$ and $50 \%$ of its nitrogen is translocated to the cane plant. It may be estimated that, under field conditions, $6-12 \mathrm{~kg}^{-\mathrm{ha}^{-1}} \mathrm{can}$ be translocated from the original seedpieces to the cane plant (shoots and roots). These values can represent $5-10 \%$ of total $\mathrm{N}$ required by the cane plant.
\end{abstract}

Key Words: $\mathrm{N}$ translocation;is $\mathrm{N}$; $\mathrm{N}$ from seedpiece reserve; sugarcane; cane plant

\section{INTRODUÇÃO}

Apesar de o nitrogênio contribuir com um porcento, em média, na massa seca total da canade-açúcar, seu papel é tão importante quanto ao do carbono, hidrogênio e oxigênio que constituem juntos mais de $90 \%$ da matéria seca. No solo, o nitro- gênio disponível às plantas é suprido pela mineralização da matéria orgânica, fixação biológica e adição de fertilizantes nitrogenados. Estudos recentes tem demonstrado que a fixação biológica do $\mathrm{N}_{2}$ contribui significativamente no nitrogênio total da cultura da cana-de-açúcar, podendo bactérias fixadoras como Acetobacter diazotrophicus locali

\footnotetext{
'Parte da Dissertação de Mestrado do primeiro autor. ${ }^{2}$ Bolsista do CNPq.
}

Sci. agric., Piracicaba, 52(2):199-209, mai./ago. 1995 
zarem-se tanto na rizosfera, como internamente a planta, em tecidos de raízes, colmo e folhas (BODDEY et al., 1991; URQUIAGA \& DÖBEREINER, 1991; URQULAGA et al., 1992; REIS et al., 1994). A água de irrigação ou de chuva contém quantidade variável de nitrogénio mineral que pode ser absorvido pelas raizes e folhas. Outra fonte de nitrogênio é a amônia da atmosfera que pode ser absorvida pela folhagem. Deve-se tambem considerar como fonte de nitrogenio, no ciclo de cana-planta, o nutriente contido no tolete de plantio (colmo-semente), e para soqueiras a reserva em rizomas ou colmos subterraneos (parte basal do colmo que permanece enterrado no solo após o corte da cana) e raízes.

Poucos trabalhos relacionados à redistribuição da reserva de nitrogénio de sementes podem ser encontrados na literatura. JENSEN et al. (1985), usando sementes de ervilha, feijoeiro e cevada marcadas com ${ }^{15} \mathrm{~N}$, determinaram que cerca de $95 \%$ do $\mathbf{N}$ original da semente translocou-se a parte aérea e raízes das plantas, após duas semanas da germinação.

Embora seja amplamente aceito que quanto mais bem nutridos estiverem, no plantio, os toletes de cana-de-açúcar melhor será a brotação e desenvolvimento inicial da cana-planta, pouco se conhece a respeito da utilização da reserva orgânica e redistribuição de nutrientes do tolete. Na literatura verificam-se trabalhos que demonstraram resposta à pré-fertilização com nitrogenio, alguns meses antes do corte de colmos destinados ao plantio, e menções sobre a influência do nitrogênio de colmossemente na brotação da cana-de-açúcar (HUMBERT, 1968; CASAGRANDE, 1991).

BACCHI (1983) e CASAGRANDE (1991) afirmaram que nos primeiros 30 dias quando ocorre a emissão de rázes de fixação e brotação de gemas, a cana-planta vive da reserva de nutrientes do tolete, que é redistribuida, e parcialmente dos nutrientes absorvidos pelas raizes de fixação. Após esse período, segundo os autores, inicia-se o desenvolvimento das raízes dos perfilhos primários, depois dos secundários, e assim sucessivamente, sendo que as rázes de fixação vão perdendo a função, passando a cana-planta a depender exclusivamente da atividade das raizes dos perfilhos.

Com referéncia a redistribuição da reserva nitrogenada na cana-de-açúcar, TAKAHASHI (1960) determinou que o nitrogennio $\left({ }^{13} \mathrm{~N}\right)$ absorvido da urtia aplicada ds folhas e que se acumulou no perfilho primário, ap6s tres meses redistribuin-se entre ele e novos perfilhos, na proporção de 56 e 44\%, respectivamente. TRIVELIN et al. (1984) determinaram que na primeira rebrota da cana-deaçúcar ocorreu a redistribuição do nitrogênio $\left({ }^{15} \mathrm{~N}\right)$ acumulado em rizomas e raízes, ficando a parte aérea e rázes da rebrota com partes iguais desse nitrogênio. Com relação a reserva de nitrogênio do colmo-semente, SAMPAIO et al. (1988) verificaram que toletes marcados $c 0 m{ }^{15} \mathrm{~N}$ de $15 \mathrm{~cm}$ de comprimento e dois nós, cultivados em solução nutritiva, liberaram cerca de $65 \%$ do nitrogenio da reserva ate 90 dias da emergencia das plantas, sendo que mais de $85 \%$ do $\mathrm{N}$ translocado localizou-se na parte aérea.

SIMÕES NETO \& MARCOS (1987) verificaram existir influência da quantidade e localização da reserva energética em toletes unigemares de canade-açúcar, no desenvolvimento inicial da planta. A quantidade de reserva energética em toletes mostrou exercer influência ao longo de 44 dias de crescimento do broto primário e tendeu a ser progressivamente maior, nos níveis mais elevados de reserva. O melhor desempenho foi obtido com toletes com maior reserva localizada no entrenó inferior à gema.

Uma questão não totalmente esclarecida trata-se da baixa resposta da cana-planta à adubação nitrogenada. Verifica-se da literatura falta de resposta em cerca de 70 a $80 \%$ dos ensaios com canaplanta. Para soqueiras a situação se inverte, sendo que mais de $70 \%$ dos ensaios mostraram responder positivamente. Fatores foram sugeridos para explicar a falta de resposta da cana-planta à fertilização mineral com nitrogênio, como: a fixação biológica do nitrogênio atmosférico; perdas por lixiviação de $\mathrm{N}$-fertilizante; vigor do sistema radicular da canaplanta comparada a de soqueiras; maior disponibilidade de $\mathbf{N}$-mineral no solo após a reforma do canavial, influenciado pelo preparo mecânico, calagem, adubação e incorporação de restos da cultura anterior; além de condições climáticas como temperatura e pluviosidade (ALBUQUERQUE \& MARINHO, 1983; ZAMBELLO \& AZEREDO, 1983; AZEREDO et al., 1986; BITTENCOURT et al., 1986; CARNAUBA, 1990; BODDEY et al., 1991; URQUIAGA \& DÖBEREINER, 1991; URQUIAGA et al., 1992).

O presente trabalho objetivou avaliar, em ensaio de vaso, o aproveitamento da reserva orghnica e de nitrogenio $\left({ }^{15} \mathrm{~N}\right.$ ) do tolete de plantio (colmo-semente) nos estádios iniciais de desenvolvimento da cana-planta. 


\section{MATERIAL E METODOS}

Os toletes para o plantio (colmos-semente) marcados com ${ }^{15} \mathrm{~N}$, contendo um nó (uma gema) mais metade do entre-nó inferior e metade do superior, foram obtidos do terço médio de colmos colhidos em ensaio prévio em campo, com a variedade NA-5679, sendo o solo fertilizado com uréia enriquecida no isótopo pesado de nitrogênio. A colheita deu-se aos 10 meses do ciclo de cana-planta.

Um tolete foi selecionado ao acaso como amostra inicial dentre os obtidos de cada colmo, e nele foram feitas determinações de umidade, teor de $\mathrm{N}$ e abundância isotópica do ${ }^{15} \mathrm{~N}$. Nos demais tomaram-se a massa fresca, sendo estimada a massa seca, conteúdo de $\mathrm{N}$ e abundância isotópica de ${ }^{15} \mathrm{~N}$ (átomos \%), considerando-se os correspondentes valores da amostra inicial.

Os toletes foram plantados em vasos com capacidade para $6 \mathrm{~kg}$ de solo (um por vaso) sendo cobertos com uma camada de $5 \mathrm{~cm}$ de terra. A terra foi colhida na camada de 0 a $30 \mathrm{~cm}$ de uma Terra Roxa Estruturada Latossólica, correspondendo a RHODIC KANHAPLUDALF na classificação Americana (Torrado*, 1989, citado por DOURADO NETO, 1989). A terra foi tamizada em peneira de $2 \mathrm{~mm}$ de malha e apresentou as seguintes características químicas: 5,7 para pH em água (2:1); 13,4 $\mathrm{mgdm}^{-3}$ de $\mathrm{P},\left(\mathrm{H}_{2} \mathrm{SO}_{4} 0,05 \mathrm{~N}\right) ; \mathrm{K}^{+}, \mathrm{Ca}^{2+}, \mathrm{Mg}^{2+}$, $\mathrm{H}^{+}+\mathrm{Al}^{3+}: 0,47,4,10,0,92,6,40 \mathrm{cmol}^{3} \mathrm{~kg}^{-1}$ respectivamente; e teor de carbono orgânico $(C \%)$ de 0,73\% (análise realizada no Laboratorio do Departamento de Solos, Geologia e Fertilizantes da ESALQ/USP, Piracicaba, SP). Previamente ao plantio procedeu-se a correção da terra, aplicando-se carbonato de cálcio em dose equivalente a $1,25 \mathrm{cmol}$ de Ca. $\mathrm{kg}^{-1}$ de solo. No plantio foi feita a fertilização da terra, aplicando-se $25 \mathrm{mg}$ de N. $\mathrm{kg}^{-1}$ de solo e $83 \mathrm{mg}$ de $P_{2} O_{3} . \mathrm{kg}^{-1}$ de solo com sulfato de amônio $e$ superfosfato triplo, respectivamente. Periodicamente foi procedida a reposição de água aos vasos, a fim de atingir $80 \%$ da capacidade de retenção do solo.

O experimento foi conduzido em casa de vegetação no Centro de Energia Nuclear na Agricultura (CENA), Piracicaba, SP, no período de jutho de 1984 a abril de 1985.

A emergência do broto primário dos toletes ocorreu no período entre o $12^{\circ}$ e $29^{\circ}$ dia após o plantio (dap). As médias de temperaturas máxima e mínima, dos 30 dias seguintes ao plantio, foram respectivamente de 28,0 e $11,1^{\circ} \mathrm{C}$ (dados forneci-

*Torrado, P.V., Comunicação pessoal, 1989, citado por DOURADO NETO (1989). dos pelo Departamento de Física e Meteorologia, ESALQ/USP, Piracicaba, SP).

Colheitas foram realizadas em 16 tempos diferentes, do $37^{\circ}$ ao $275^{\circ}$ dia apos o plantio (dap). Em cada tempo colheram-se plantas de 4 vasos que foram subdivididas em amostras de: parte aérea, raízes e tolete remanescente. Nas amostras, após serem secas a $60^{\circ} \mathrm{C}$, em estufa ventilada, e moidas em moinho tipo Wiley (peneira com malha de $0,4 \mathrm{~mm}$ ), foram procedidas as determinações de nitrogênio total por digestão-destilação micro-Kjeldahl e de composição isotópica do nitrogênio (átomos \% de ${ }^{15} \mathrm{~N}$ ) por espectrometria de massas, segundo metodologias descritas por TRIVELIN et al. (1973).

A recuperação do nitrogênio original do tolete de plantio ( $R$; mg.vaso $^{-1}$ ) na parte aérea e raízes (N-translocado) e no tolete remanescente (N-remanescente) foi obtida com a equação (1):

$\mathrm{R}=\left[\left(\text { at. } \%{ }^{15} \mathrm{~N} \text { exc. }\right)_{\mathrm{p}} /(\text { at. } \% \text { is } \mathrm{N} \text { exc. })_{\mathrm{p}}\right]$. (N-total)

significando: (at. \% ${ }^{15} \mathrm{~N}$ exc.) - atomos \% de ${ }^{15} \mathrm{~N}$ em excesso na planta, obtido, subtraindo-se o valor de abundância natural $\left(0,367\right.$ átomos $\%$ de $\left.{ }^{15} \mathrm{~N}\right) \mathrm{da}$ concentração de ${ }^{15} \mathrm{~N}$ (átomos \%) da amostra; (at. \%

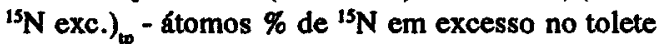
de plantio, obtido da abundância de ${ }^{15} \mathrm{~N}$ do tolete de plantio (átomos \%) subtraida do valor de abundância natural; $e$ ( $\mathrm{N}$-total) $\mathrm{p}$ o nitrogênio total na planta (mg. vaso-1).

No cálculo da recuperação porcentual (R'; \%) do nitrogênio original do tolete de plantio, em diferentes partes da cana-planta, fez-se uso da expressão (2):

$R^{\prime}=\left[R /(\text { N-total })_{\varphi}\right] \cdot 100$

onde: (N-total) ${ }_{\text {r }}$ refere-se ao nitrogênio total do tolete no plantio (mg.vaso ${ }^{-1}$ ou mg.tolete ${ }^{-1}$ ).

0 experimento foi conduzido em delineamente inteiramente casualizado, com 16 tratamentos que corresponderam aos tempos $(t)$ de: $37,53,69,85,109,123,148,161,178,195,214$, 223, 232, 248, 261, 275 dias após o plantio (dap). Cada tratamento constou de 4 repetições. Os resultados obtidos foram submetidos à análise de variância, comparando-se as médias pelo teste de Duncan ao nível de $5 \%$ de probabilidade. Utilizouse também da correlação linear simples e múltipla. Nas regressões foram apresentados os coeficientes de correlação (r) e suas significâncias estatísticas pelo teste $\mathrm{F}$ a $5 \%$ e $1 \%$ de probabilidade, representados por um e dois asteriscos respectivamente. 


\section{RESULTADOS E DISCUSSÃO}

Os resultados de massa seca, nitrogênio total e de abundância de ${ }^{15} \mathrm{~N}$ do tolete de cana no plantio $(t=0)$ e nas colheitas $(t=d a p)$ constam da TABELA 1.

A análise de variância dos resultados de massa seca e nitrogênio total do tolete no plantio $(t=0)$ evidenciou diferenças estatísticas significativas entre tratamentos, não ficando caracterizados os resultados pela aleatoriedade. Devido a esse fato, podem ocorrer distorções nas comparações entre tratamentos de grandezas absolutas como massa seca e nitrogênio total, porém, não ocorrerão entre valores relativos que normalizam os resultados dos tratamentos, como a porcentagem da massa seca do tolete à diferentes tempos $(\mathrm{t}=$ dap $)$ relativa à do plantio $(t=0)$, porcentagem de exportação do $\mathrm{N}$ original do tolete com o tempo, e a porcentagem de recuperação do $\mathrm{N}$ do tolete de plantio em diferentes orgãos da cana-planta em função do tempo.

A massa seca final do tolete $(t=d a p)$ correlacionou-se inversamente com o tempo (dap) $\left(r=-0,81^{* *}\right)$ mas não mostrou relação com a massa seca do colmo-semente no plantio $(r=0,24 n s)$. Por sua vez, a variação da massa seca do tolete ( $\triangle \mathrm{MS}$ ) mostrou relação direta com o tempo $\left(r=0,71^{* *}\right)$, evidenciando que ocorreu a degradação da reserva orgânica do tolete com o tempo após o plantio.

$O$ conteúdo de nitrogênio do tolete $(\mathrm{t}=$ dap) correlacionou-se diretamente com o tempo após o

TABELA 1. Massa seca, nitrogênio total e abundância de ${ }^{15} \mathrm{~N}$ do nitrogênio do tolete de cana no plantio $(t=0)$ e a diferentes tempos $(\mathrm{t}=$ dap) (média de quatro repetições).

\begin{tabular}{|c|c|c|c|c|c|c|c|c|}
\hline \multirow{3}{*}{$\frac{\text { Tempo }}{\text { dap }}$} & \multicolumn{3}{|c|}{ Massa Seca } & \multicolumn{3}{|c|}{ Nitrogênio Total } & \multicolumn{2}{|c|}{ Abund. de ${ }^{15} \mathrm{~N}^{1}$} \\
\hline & \multicolumn{3}{|c|}{ g.tolete ${ }^{-1}$} & \multicolumn{3}{|c|}{ mg.tolete ${ }^{-1}$} & \multicolumn{2}{|c|}{ atomos \% } \\
\hline & $t=0$ & $t=d a p$ & $\Delta^{2}$ & $t=0$ & $t=$ dap & $\Delta^{2}$ & $t=0$ & $t=$ dap \\
\hline 37 & $17,2 \mathrm{c}$ & $13,2 \mathrm{a}$ & 4,0 & $42,7 \mathrm{c}$ & $37,7 \mathrm{~b}$ & 5,0 & 0,721 & 0,623 \\
\hline 53 & $20,2 b c$ & $11,5 \mathrm{ab}$ & 8,8 & 54,9 abc & $38,1 \mathrm{~b}$ & 16,8 & 0,691 & 0,622 \\
\hline 69 & $21,1 \mathrm{bc}$ & $11,1 \mathrm{abc}$ & 10,0 & $57,5 \mathrm{abc}$ & $42,9 \mathrm{ab}$ & 14,6 & 1,030 & 0,940 \\
\hline 85 & $24,8 \mathrm{ab}$ & 13,1 a & 11,7 & $63,1 \mathrm{ab}$ & $42,8 \mathrm{ab}$ & 20,3 & 0,674 & 0,625 \\
\hline 109 & $24,7 \mathrm{ab}$ & $10,5 \mathrm{abc}$ & 14,2 & $65,7 \mathrm{ab}$ & $51,8 \mathrm{ab}$ & 13,9 & 0,630 & 0,578 \\
\hline 123 & $23,6 a b$ & $11,0 \mathrm{abc}$ & 12,6 & 69,4 a & $48,4 \mathrm{ab}$ & 21,0 & 0,676 & 0,645 \\
\hline 148 & $23,9 \mathrm{ab}$ & 9,6 bed & 14,3 & $65,1 a b$ & $49,3 \mathrm{ab}$ & 15,8 & 1,190 & 0,898 \\
\hline 161 & $20,2 \mathrm{bc}$ & 8,4 cdef & 11,9 & $51,6 b c$ & $41,3 \mathrm{~b}$ & 10,3 & 1,290 & 0,974 \\
\hline 178 & $26,7 \mathrm{a}$ & $10,8 \mathrm{abc}$ & 15,9 & $68,9 \mathrm{a}$ & $45,2 \mathrm{ab}$ & 23,7 & 0,634 & 0,529 \\
\hline 195 & $24,6 a b$ & $10,8 \mathrm{abc}$ & 13,7 & $60,5 \mathrm{ab}$ & $44,3 \mathrm{ab}$ & 16,2 & 0,737 & 0,612 \\
\hline 214 & $22,8 \mathrm{ab}$ & $9,9 \mathrm{bc}$ & 12,9 & $56,3 \mathrm{abc}$ & $54,5 \mathrm{a}$ & 1,8 & 0,956 & 0,698 \\
\hline 223 & $23,8 \mathrm{ab}$ & 8,7 bcdef & 15,1 & $67,7 \mathrm{ab}$ & $49,4 \mathrm{ab}$ & 18,3 & 1,163 & 0,918 \\
\hline 232 & $17,2 \mathrm{c}$ & $6,4 \mathrm{f}$ & 10,8 & 51,9 bc & $41,8 \mathrm{ab}$ & 10,1 & 1,033 & 0,672 \\
\hline 248 & $21,0 \mathrm{bc}$ & $7,0 \mathrm{def}$ & 14,0 & $57,9 \mathrm{abc}$ & $42,9 \mathrm{ab}$ & 15,0 & 1,020 & 0,833 \\
\hline 261 & 20,5 bc & 6,6 ef & 13,9 & $53,8 \mathrm{abc}$ & $49,9 \mathrm{ab}$ & 3,9 & 0,826 & 0,575 \\
\hline 275 & $25,9 \mathrm{a}$ & 9,3 bcde & 16,6 & $65,9 \mathrm{ab}$ & $54,4 \mathrm{a}$ & 11,5 & 0,867 & 0,810 \\
\hline
\end{tabular}

' Média obtida da pondera ção dos resultados de $\mathrm{N}$-total e de abundância de ${ }^{15} \mathrm{~N}$, consideradas as 4 repetições.

${ }^{2} \Delta$ significa a diferença entre as médias de massa seca ou nitrogênio total do tolete no início $(t=0)$ e no final $(\mathrm{t}=$ dap).

Médias nas colunas seguidas de letras distintas diferem significativamente pelo teste de Duncan $(p=0.05)$. 
plantio $\left(r=0,54^{*}\right)$ e com o nitrogênio total do tolete no plantio $\left(r=0,58^{*}\right)$. A redução do $N$ total do tolete $(\Delta N$-total) não evidenciou relação com o tempo (dap) $(r=-0,22 n s)$. Esses resultados, por si, não evidenciam se ocorreu a liberação de nitrogênio do tolete de plantio para o desenvolvimento inicial da cana-planta.

Os resultados de abundância de ${ }^{15} \mathrm{~N}$ do nitrogênio do tolete no plantio $(t=0)$ e nas colheitas
( $\mathrm{t}=$ dap) que constam da TABELA 1, revelaram que ocorreu significativa diluição isotópica do conteúdo inicial de nitrogênio. A despeito de as abundâncias de ${ }^{15} \mathrm{~N}$ dos toletes no plantio terem sido obtidas indiretamente e os valores à diferentes tempos $(t=$ dap) tratarem-se de medidas diretas, a redução das abundâncias em todos os tratamentos possibilita afirmar que ocorreu significativa incorporação de nitrogênio, com concentração natural de ${ }^{15} \mathrm{~N}$, no tolete. $\mathrm{O}$ mesmo efeito no nitrogênio do tolete foi verificado

TABELA 2. Conteúdo de nitrogênio do tolete (N-total), recuperação do $\mathrm{N}$ original do tolete de plantio, nitrogênio incorporado ao tolete ( $\mathrm{N}$-incorporado), e nitrogênio exportado do tolete de plantio ( $\mathrm{N}$ original exportado do tolete) à diferentes tempos-dap (média de quatro repetições).

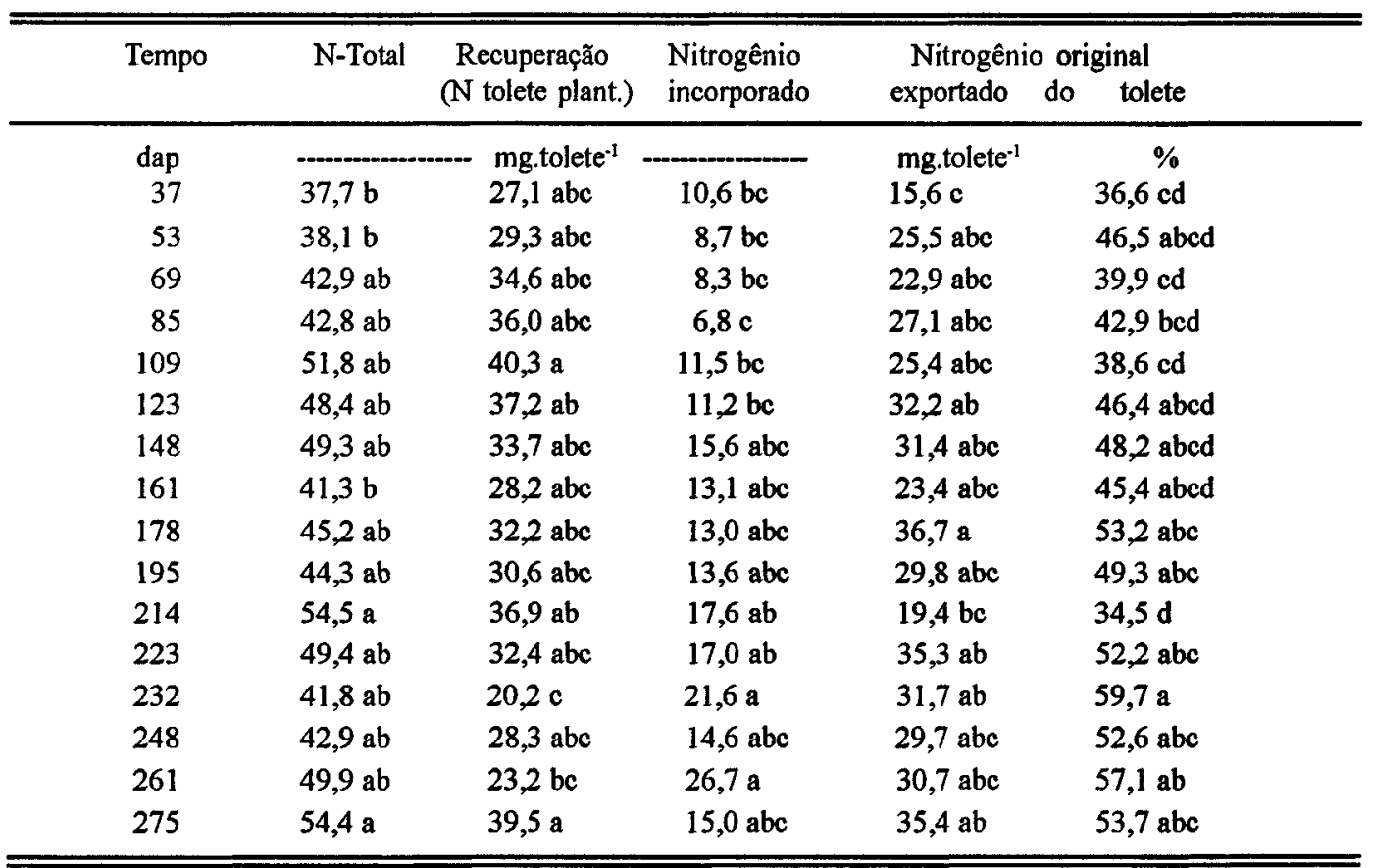

Médias nas colunas seguidas de letras distintas diferem significativamente pelo teste de Duncan $(p=0.05)$.

por SAMPAIO et al. (1988), em ensaio com solução nutritiva sem $\mathrm{N}$, que sugeriram, naquela condição experimental, a ocorrência de fixação microbiológica do $\mathrm{N}$ atmosférico nas raízes das plantas.

LIMA et al. (1987) e URQUIAGA et al. (1992), fazendo crescer diferentes variedades e espécies de cana em solo marcado com ${ }^{15} \mathrm{~N}$, constataram significativa redução na abundância do isótopo em alguns cultivares de Saccharum spp, e estimaram contribuições devido a fixação biológica de $\mathbf{3 0}$ a $60 \%$ no $\mathrm{N}$-total acumulado pelas plantas.Como no presente trabalho não se efetuou o balanço isotópico nitrogenado no sistema solo-planta, a fim de estimar a possível contribuição da fixação biol6gica, doravante, o conteúdo de nitrogênio na planta oriundo de fontes que não da reserva original do tolete (o $\mathrm{N}$ do solo absorvido pelas raízes e o da atmosfera fixado por ação de microrganismos) será referido como nitrogênio incorporado.

Na TABELA 2 constam os resultados, à diferentes tempos (dap), de nitrogênio total no tolete, 
incluido o $\mathrm{N}$ incorporado, a recuperação do $\mathrm{N}$ original do tolete de plantio ( $\mathrm{N}$ do tolete de plantio), o $\mathrm{N}$ incorporado no tolete, e a quantidade e porcentagem do $\mathrm{N}$ original do tolete de plantio exportado. O nitrogênio exportado do tolete de plantio não foi obtido da simples diferença entre os conteúdos de nitrogênio inicial $(t=0)$ e final $(t=$ dap), visto que ocorreu a incorporação de nitrogênio, como sugeriram os resultados de abundância de ${ }^{13} \mathrm{~N}$.

$O$ nitrogênio exportado, à cada tempo, foi obtido da diferença entre o conteúdo de nitrogênio do tolete no plantio (TABELA 1) e o $\mathrm{N}$ original recuperado do tolete de plantio (TABELA 2), calculado com os resultados de concentração de ${ }^{15} \mathrm{~N}$ e de $\mathrm{N}$-total (equação 1). $\mathrm{O} \mathrm{N}$ incorporado a cada tempo $(t=$ dap), dado à TABELA 2 , refere-se a diferença entre o conteúdo de $\mathrm{N}$-total do tolete e o valor correspondente ao $\mathrm{N}$ recuperado do tolete de plantio. Dessa forma, deve ser entendido por nitrogênio exportado o liberado do tolete e relativo ao conteúdo inicial $(t=0)$. Esse nitrogênio deve ter-se translocado aos novos tecidos da cana-planta: parte aérea (folhas e colmo) e raizes.

Os resultados do nitrogênio exportado do tolete (mg.tolete ${ }^{-1}$ ) revelaram que a redistribuição do nutriente foi função direta do tempo $\left(r=0,58^{*}\right)$ e do conteúdo de $\mathrm{N}$ do tolete no plantio $\left(\mathrm{r}=0,72^{* *}\right)$. A relação do nitrogênio exportado $\left(\mathrm{N}_{\text {exp }}\right.$ ) com o tempo (t) e a reserva inicial de $\mathrm{N}$ do tolete $\left(\mathrm{N}_{\mathrm{t}=0}\right)$ está representada na equação (3).

$N_{\text {exp }}=0,034 \cdot t+0,38 \cdot N_{t=0} \quad(r=0,83 * *)$

Os resultados acima permitem afirmar que toletes com maior reserva de nitrogênio no plantio $\left(\mathrm{N}_{t=0}\right)$ foram os que liberaram maior quantidade do nutriente, podendo esse efeito relacionar-se com a resposta na brotação e desenvolvimento inicial da cana-planta de mudas pre-fertilizadas com nitrogênio, como mencionado por HUMBERT (1968).

O nitrogênio da reserva do tolete foi exportado em função do tempo após o plantio, como já observado dos resultados à TABELA 2, estando o fenômeno ilustrado na Figura 1. Da Figura 1 podese estimar que cerca de $20 \%$ da reserva exportável de $\mathrm{N}$ do tolete, ou seja, $10 \%$ do $\mathrm{N}$ da reserva original, translocou-se aos novos tecidos por volta de 20dap, portanto, à época (tempo médio) da emergência do broto primário. Aos 50-60dap, cerca de $40 \%$ do $\mathrm{N}$ original do tolete já havia sido exportado (aproximadamente $80 \%$ do $\mathrm{N}$ exportável); decorridos 5 a 6 meses do plantio $50 \%$ do $\mathrm{N}$ original do

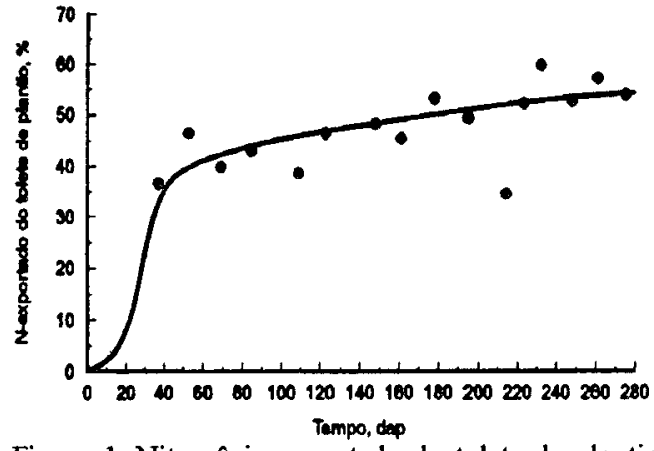

Figura 1. Nitrogênio exportado do tolete de plantio em função do tempo-dap.

tolete foi exportado (praticamente $100 \%$ do $\mathrm{N}$-ex portável). Fica claro, portanto, que ocorreram nos 50-60 dias seguintes ao plantio, as maiores taxas de translocação do $\mathrm{N}$ do tolete, como suporte à emergêncía e ao desenvolvimento inicial de raízes de fixação e do perfilho primário.

Mesmo sendo o tolete uma fonte de $\mathrm{N}$ para o desenvolvimento inicial da cana-planta, a ele foi incorporado nitrogênio externo ( $\mathrm{N}$ do solo e da fixação biológica) como função direta do tempo $(r=0,79 * *)$. O N incorporado não mostrou relação com o nitrogênio exportado $(r=0,33 n s)$. Esses resultados colocam em evidência que o tolete é parte integrante da planta de cana-de-açúcar, mostrando-se metabolicamente ativo e participando como fonte e dreno de nitrogênio e provavelmente de outros nutrientes.

Os resultados de massa seca e nitrogênio total na cana-planta (raízes, parte aérea e planta toda) à diferentes tempos do plantio constam da TABELA 3. Na TABELA 4 são apresentados os resultados de recuperação do $\mathrm{N}$ original do tolete na canaplanta, em função do tempo (dap), obtidos com os dados de abundância de ${ }^{15} \mathrm{~N}$ (equações $1 \mathrm{e} 2$ ).

A massa seca de toletes diminuiu com o tempo (TABELA 1), significando que a energia produzida na degradação da reserva orgânica dos toletes foi utilizada no desenvolvimento inicial de novos tecidos da cana-planta. Dos resultados das TABELA 1 e 3 pode-se verificar que a redução da matéria seca do tolete ( $\triangle M S$ ) somente foi compensada pela produção de massa seca de novos tecidos (raízes e parte aérea) aos 85dap. Isso evidencia que nos estádios iniciais a taxa de respiração foi maior do que a de fotossíntese, ficando a emissão e crescimento inicial de raízes e do perfílho primário na dependência direta da reserva orgânica do tolete. 
TABELA 3. Massa seca e nitrogênio total em raízes, parte aérea e planta toda de cana-de-açúcar à diferentes tempos do plantio - dap (média de quatro répetições).

\begin{tabular}{|c|c|c|c|c|c|c|}
\hline \multirow[t]{2}{*}{ Tempo } & \multicolumn{6}{|c|}{ Massa Seca } \\
\hline & Raízes & Parte aérea & Planta toda ${ }^{1}$ & Raizes & Parte aérea & Planta toda 1 \\
\hline dap & \multicolumn{3}{|c|}{ ב__naso ${ }^{-1}$ - } & \multicolumn{2}{|c|}{ mg.vaso ${ }^{-1}$} & - \\
\hline 37 & $0,18 \mathrm{~g}$ & $1,29 \mathrm{e}$ & $14,7 \mathrm{e}$ & $2,2 \mathrm{~g}$ & $21,5 \mathrm{j}$ & $61,4 i$ \\
\hline 53 & $0,99 \mathrm{fg}$ & $3,25 \mathrm{e}$ & $15,7 \mathrm{e}$ & $13,5 \mathrm{fg}$ & $51,5 \mathrm{ij}$ & $103,1 \mathrm{hi}$ \\
\hline 69 & $1,77 \mathrm{fg}$ & $4,75 \mathrm{e}$ & $17,6 \mathrm{e}$ & $14,0 \mathrm{fg}$ & $67,9 \mathrm{hij}$ & $124,9 \mathrm{hi}$ \\
\hline 85 & 5,37 efg & $13,2 \mathrm{e}$ & $31,7 \mathrm{e}$ & 40,0 def & $117,2 \mathrm{ghi}$ & $200,0 \mathrm{fgh}$ \\
\hline 109 & 7,16 def & $15,0 \mathrm{e}$ & $32,6 \mathrm{e}$ & $33,6 \mathrm{efg}$ & $141,1 \mathrm{fgh}$ & $226,5 \mathrm{fg}$ \\
\hline 123 & 7,74 def & $26,0 \mathrm{de}$ & $44,7 \mathrm{de}$ & 47,2 def & 181,7 efg & 265,5 ef \\
\hline 148 & 13,5 bed & $42,5 \mathrm{~d}$ & $65,6 \mathrm{~cd}$ & $63,0 \mathrm{de}$ & $247,8 \mathrm{de}$ & 360,9 de \\
\hline 161 & 25,4 a & $39,6 \mathrm{~d}$ & $73,3 \mathrm{c}$ & $109,5 \mathrm{ab}$ & 226,0 def & $376,8 \mathrm{~cd}$ \\
\hline 178 & 17,3 bc & 82,7 bc & $110,8 \mathrm{ab}$ & $72,6 \mathrm{~cd}$ & 354,3 bc & 472,1 bc \\
\hline 195 & $12,0 \mathrm{~cd}$ & $68,3 \mathrm{c}$ & 91,2 bc & $60,4 \mathrm{de}$ & 269,5 cde & $374,2 \mathrm{~cd}$ \\
\hline 214 & 15,8 bc & 94,7 ab & $120,6 \mathrm{ab}$ & $55,9 \mathrm{de}$ & $313,0 \mathrm{bcd}$ & 423,4 bcd \\
\hline 223 & $12,7 \mathrm{bcd}$ & $110,5 \mathrm{a}$ & $129,1 \mathrm{a}$ & 77,4 bcd & $383,8 \mathrm{~b}$ & 478,1 bc \\
\hline 232 & 16,1 bc & 88,7 abc & $111,1 \mathrm{ab}$ & 127,1 a & $491,8 \mathrm{a}$ & $644,1 \mathrm{a}$ \\
\hline 248 & 11,3 cde & 108,8 a & $127,0 \mathrm{a}$ & $72,4 \mathrm{~cd}$ & $550,9 a$ & $666,2 a$ \\
\hline 261 & 15,9 bc & $95,8 \mathrm{ab}$ & $118,3 \mathrm{ab}$ & $118,8 \mathrm{a}$ & 516,5 a & $685,0 \mathrm{a}$ \\
\hline 275 & 19,0 b & $99,2 \mathrm{ab}$ & $127,5 \mathrm{a}$ & $104,2 \mathrm{abc}$ & $369,5 \mathrm{~b}$ & $528,1 \mathrm{~b}$ \\
\hline
\end{tabular}

1 Planta toda $=$ raízes + parte aérea + tolete

Médias nas colunas seguidas de letras distintas diferem significativamente pelo teste de Duncan $(p=0.05)$.

SIMÕES NETO \& MARCOS (1987) verificaram existir influência direta da quantidade de reserva orgânica do tolete, no tempo de brotação e no desenvolvimento inicial da cana-planta. SAMPAIO et al. (1988) determinaram que aos 30 e 60 dias após a emergência, a formação de materia nova compensou as perdas de massa seca do tolete, para plantas crescidas em solução nutritiva, respectivamente, com e sem nitrogênio.

Valores porcentuais da variação, com o tempo, da matéria seca dos toletes (Figura 2) indicam que a taxa de degradação da reserva orgânica foi elevada até por volta de 60-70dap, estabilizando-se com baixas taxas mas crescentes, a partir de 7080dap. Esse estádio é coincidente àquele em que a massa seca de novos tecidos se iguala à redução de massa dos toletes (TABELAS 1 e 3). Mais de 50\% da reserva orgânica inicial foi degradada até por volta dos 6 meses do plantio. A grandeza desses valores são de mesma ordem que à do $\mathbf{N}$ exportado do tolete, possibilitando inferir que a degradação da reserva orgânica do colmo-semente liberou quan-
O nitrogênio original exportado do tolete (TABELA 2) correlacionou-se diretamente com o nitrogênio total nas raízes $\left(r=0,56^{*}\right)$ e com o Ntotal da parte aérea $(r=0,59 *)$, considerados todos os tempos após o plantio (TABELA 3). Por sua vez, a redução no conteúdo de nitrogênio do tolete (TABELA 1) não evidenciou correlação significativa com o tempo ( $r=0,22 n s)$, nem tampouco com o Ntotal dos novos tecidos da cana-planta $(r=0,17 n s)$. Estes resultados colocam em evidência a utilidade da técnica isotópica com ${ }^{15} \mathrm{~N}$ na quantificação da redistribuição do $\mathrm{N}$ da reserva do tolete de plantio.

$O$ nitrogênio recuperado do tolete de plantio em novos tecidos, aos 37dap (12,97mg.vaso $\left.{ }^{-1}\right)$, repre sentou $55 \%$ do $\mathrm{N}$-total $\left(23,7 \mathrm{mg}^{\text {vaso }}{ }^{-1}\right)$ (TABELAS 3 e 4), sendo o restante $45 \%$ (10,7mg.vas $\left.\sigma^{\prime}\right)$ devido ao $\mathrm{N}$ incorporado. Após 53, 69 e 85dap, o $\mathrm{N}$ original do colmo-semente representou 27,23 e $16 \%$, respectivamente do $\mathrm{N}$-total da parte aérea e raízes da cana-planta; após 148dap o $\mathrm{N}$ derivado do tolete foi de apenas $6 \%$ do total. Assim, verifica-se acentuada redução, com o tempo, na participação 
TABELA 4. Nitrogênio do tolete de plantio (mg.vaso ${ }^{-1} \mathrm{e} \%$ ) recuperado em raízes, parte aérea e planta toda de cana-de-açúcar à diferentes tempos do plantio - dap (média de quatro repetições).

\begin{tabular}{|c|c|c|c|c|c|c|}
\hline \multirow{2}{*}{$\frac{\text { Tempo }}{\text { dap }}$} & \multicolumn{2}{|c|}{ Raízes } & \multicolumn{2}{|c|}{ Parte aćrea } & \multicolumn{2}{|c|}{ Planta toda ${ }^{1}$} \\
\hline & mg.vaso $^{-1}$ & $\%$ & mg.vaso $^{-1}$ & $\%$ & mg.vaso-1 & $\%$ \\
\hline 37 & $1,07 \mathrm{~cd}$ & 2,5 & $11,9 \mathrm{~cd}$ & 28,0 & 40,1 e & 93,8 \\
\hline 53 & $3,53 \mathrm{bcd}$ & 6,3 & 14,2 bcd & 25,9 & 47,1 cde & 86,6 \\
\hline 69 & $3,45 \mathrm{bcd}$ & 6,0 & $15,7 \mathrm{bcd}$ & 27,3 & $53,7 \mathrm{bcd}$ & 93,7 \\
\hline 85 & $4,73 a b$ & 7,4 & $19,8 \mathrm{ab}$ & 31,6 & $60,5 \mathrm{bcd}$ & 96,1 \\
\hline 109 & 3,12 bcd & 5,0 & $12,8 \mathrm{bcd}$ & 19,5 & 56,3 bcd & 85,7 \\
\hline 123 & $5,56 \mathrm{a}$ & 8,5 & $24,8 \mathrm{a}$ & 35,2 & $67,6 \mathrm{a}$ & 97,4 \\
\hline 148 & 4,61 bcd & 7,2 & 14,4 bcd & 22,7 & 52,8 cde & 82,0 \\
\hline 161 & $5,50 \mathrm{~cd}$ & 11,1 & $11,0 \mathrm{~cd}$ & 22,0 & 44,7 cde & 87,7 \\
\hline 178 & $5,05 \mathrm{a}$ & 7,4 & $26,1 \mathrm{a}$ & 37,8 & $63,4 \mathrm{ab}$ & 91,7 \\
\hline 195 & $5,24 \mathrm{abc}$ & 8,6 & $19,3 \mathrm{abc}$ & $.32,0$ & 55,2 bcd & 91,6 \\
\hline 214 & $2,69 \mathrm{bcd}$ & 4,7 & 16,0 bcd & 28,0 & 55,6 cde & 98,4 \\
\hline 223 & $4,48 \mathrm{ab}$ & 6,5 & $18,3 \mathrm{ab}$ & 26,5 & $55,2 \mathrm{abc}$ & 81,4 \\
\hline 232 & 4,26 bc & 8,9 & $15,1 \mathrm{bcd}$ & 29,9 & $39,5 \mathrm{bcd}$ & 76,1 \\
\hline 248 & $1,94 \mathrm{bcd}$ & 3,7 & 15,9 bed & 27,6 & 46,1 cde & 80,7 \\
\hline 261 & $4,29 \mathrm{bcd}$ & 7,7 & $11,7 \mathrm{~cd}$ & 21,3 & 39,2 de & 72,4 \\
\hline 275 & 4,73 bcd & 7,2 & $13,6 \mathrm{bcd}$ & 21,5 & $57,7 \mathrm{cde}$ & 87,5 \\
\hline
\end{tabular}

${ }^{1}$ Planta toda $=$ raízes + parte aérea + tolete

Médias nas colunas seguidas de letras distintas diferem significativamente pelo teste de Duncan $(p=0.05)$.

do $\mathrm{N}$ da reserva do tolete de plantio no $\mathrm{N}$-total em novos tecidos, como também está ilustrado na Figura 3. Novamente fica evidente que a contribuição fundamental do $\mathrm{N}$-tolete na nutrição nitrogenada da cana-planta ocorreu nos estádios iniciais de desenvolvimento. Dos resultados do $\mathrm{N}$ do tolete de

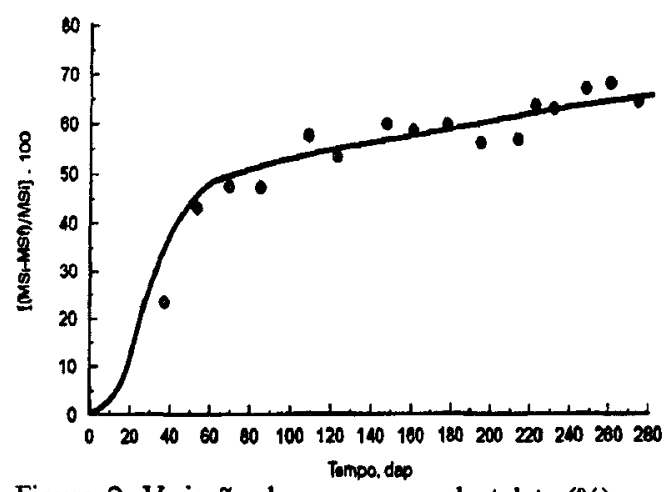

Figura 2. Variação da massa seca do tolete (\%) em função do tempo após o plantio-dap (MSi e MSf significam massa seca do tolete para $t=0$ e $t=$ dap, respectivamente). plantio translocado à cana-planta (TABELA 4), podese também verificar que, em média, mais de $80 \%$ foi recuperado na parte aérea. Mesmo em se considerando a limitação no crescimento de plantas de cana-de-açucar em vaso, os resultados de acúmulo de $\mathrm{N}$ e de redistribuição da reserva do nutriente do tolete obtidos até por volta dos 80dap podem ser equiparados aos que se verificam em campo, devido o pequeno crescimento das plantas e uma vez que elas se desenvolvem, nesse período, as expensas da reserva orgânica do tolete. Assim posto, fica evidente que o $\mathrm{N}$ da reserva do tolete é fundamental para o desenvolvimento inicial da cana-planta, no período que vai do plantio até 30-40dias após a emergência do perfilho primário, nas condições do presente trabalho de 50-60dap, confirmando as afirmativas de BACCHI (1983) e CASAGRANDE (1991), no caso específico do nutriente nitrogênio.

Os resultados de recuperação do $\mathrm{N}$ do tolete de plantio na planta toda (TABELA 4) revelaram para todos os tempos valores inferiores a $100 \%$. Independentemente de terem ocorridos erros analíticos, alguns fatores podem explicar esses resulta- 
dos, como: perdas de $\mathrm{N}$ por exsudação radicular, morte de raízes e descamação de tecidos do tolete, como também, perda de raízes junto ao material de solo nas colheitas. A tendência de queda na recuperação do $\mathrm{N}$-tolete de plantio (\%), na parte aérea e planta toda, pode ainda ser devida à perdas por volatilização de compostos nitrogenados na corrente transpiratória, que ocorre principalmente em folhas na maturidade e na senescência (STUTTE et al., 1979; SILVA \& STUTTE, 1981).

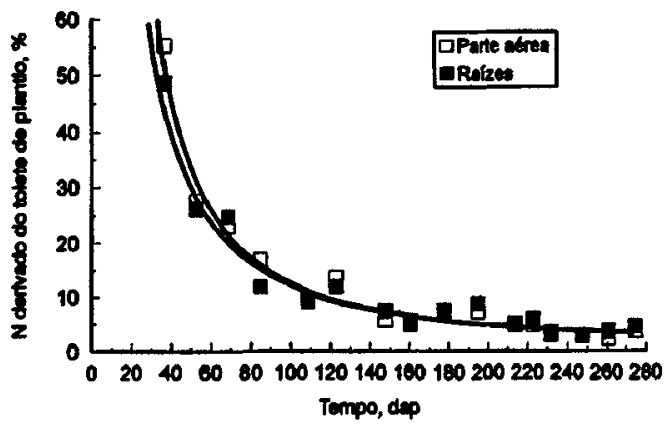

Figura 3. Nitrogênio na parte aérea e raízes (\%) derivado do $\mathbf{N}$ original do tolete de plantio em função do tempo-dap.

No plantio da cana-de-açúcar (espaçamento de $1,40 \mathrm{~m}$ entre linhas), no estado de São Paulo, são colocadas duas "canas" inteiras justapostas e cruzadas (pé com ponta) no fundo do sulco, média de 15 gemas por metro linear, o que resulta em $10 t$ ha $^{1}$ de colmos destinados ao plantio (COLETI, 1987; STOLF \& BARBOSA, 1990 e 1991). Para colmos com conteúdo de água de $70 \%$, a reserva de $\mathrm{N}$ das mudas será de 12 a $24 \mathrm{~kg} \cdot \mathrm{ha}^{-1}$, considerado os teores de nitrogênio de colmos, para cana-planta com idades variando de 10 a 14 meses, respectivamente na faixa 0,4 a $0,8 \%$ de $N$ (HAAG et al., 1987). Com base nos resultados do presente trabalho, podese estimar que $5-10 \mathrm{~kg}$ ha $^{-1}$ de nitrogênio dos toletes de plantio (liberação de cerca de $40 \%$ do $\mathrm{N}$-tolete) são translocados à novos tecidos da cana-planta até 50-60dap (30-40dae). Pode-se também estimar que até os 6 meses do plantio, $6-12 \mathrm{~kg} \cdot \mathrm{ha}^{-1}$ do $\mathrm{N}$-tolete transloca-se à novos tecidos ( $50 \%$ do $\mathrm{N}$-tolete), valores estes que podem representar 5-10\% das necessidades de $\mathbf{N}$ da cultura.

A produtividade final da cana-de-açúcar, quer seja cana-planta ou soqueira, está relacionada a fatores ambientais, fisiológicos e de manejo da cultura, sendo citado na literatura, entre outros: a temperatura, pluviosidade, tipo de solo, características genéticas da variedade, ocorrência de pragas e doenças, correção e fertilização mineral do solo, disponibilidade de nutrientes no solo, e recentemente a fixação biológica. Como a reserva orgânica e de nitrogênio do colmo-semente exerce influência direta na brotação e desenvolvimento inicial da canaplanta, deve a mesma passar a ser considerada um fator que, associado a vários outros, poderá influir na produtividade final da cana-planta.

\section{CONCLUSÕES}

1. As maiores taxas de exportação do $\mathrm{N}$ da reserva do tolete ocorreram nos 50-60 dias seguintes ao plantio (dap), sendo redistribuido nesse periodo cerca de $40 \%$ do $\mathrm{N}$ original (aproximadamente $80 \%$ do $\mathrm{N}$ exportável).

2. $50 \%$ do $\mathrm{N}$ original do tolete foi exportado até os 5 a 6 meses do plantio.

3. Intensa degradação da reserva orgânica do tolete ocorreu nos 60-70dap.

4. A redução da matéria seca do tolete somente foi compensada pela produção de massa de novos tecidos (raizes e parte aérea) por volta dos 3 meses do plantio.

5. Mais de $50 \%$ da reserva orgânica inicial foi degradada até por volta dos 6 meses do plantio.

6. Em condições de campo, cerca de 5-10kg.ha-1 de nitrogénio dos toletes de plantio pode ser translocado à novos tecidos da cana-planta até 50-60dap (3040dae).

7. 6-12 kg.ha-1 do N-tolete transloca-se aos novos tecidos da cana-planta, até os 6 meses do plantio, podendo representar até 5-10\% das necessidades de $\mathbf{N}$ da cultura.

8. A reserva orgânica e de nitrogênio do colmosemente influencia diretamente na brotação e desenvolvimento inicial da planta, devendo ser considerada um fator, associado a vários outros, a influir na produtividade da cana-planta.

\section{REFERÊNCIAS BIBLIOGRÁFICAS}

ALBUQUERQUE, G.A. C.; MARINHO, .L. Adubação na região norte-nordeste. In: ORLANDO Fo., J. Nutrição e adubação da cana-de-açúcar no Brasil. Piracicaba, IAA Planalsucar, 1983 . p. 267-286. 
AZEREDO, D.F.; BOLSANELLO, J.; EBER, H.; VIEIRA, J.R. Nitrogenio em cana-planta doses e fracionamento. STAB-Afficar, Alcool e Subprodutos, Piracicaba, v.4, n.5, p.25-29, 1986.

BACCHI, O. O. S. Botanica da cana-de-açúcar. In: ORLANDO Fo., J. Nutriçio e adubaçio da canade-aftcar no Brasil. Piracicaba, IAA Planalsucar, 1983. p.25-37.

BITTENCOURT, V.C.; FAGANELLO, B.F.; SALATA, J.C. Eficiência da adubação nitrogenada em cana-deaçúcar (planta). TAB - Afficar, Álcool e Subprodutos, Piracicaba, v.5, n.1, p.26-33, 1986.

BODDEY, R.M.; URQUIAGA, S.; REIS, V.; DÖBEREINER, J. Biological nitrogen fixation associated with sugar cane. Plant and Soil, The Hague, v.137, n.1, p. 111-117, 1991.

CARNAUBA, B.A.A. O nitrogênio e a cana-de-açúcar. STAB-Agficar, Alcool e Subprodutos, Piracicaba, v.8, n.3/4, p.24-41, 1990.

CASAGRANDE, A.A. TÓpicos de morfologia e fisiologia da cana-de-agficar. Jaboticabal, FUNEP, 1991, $157 \mathrm{p}$.

COLETI, J.T. Técnica cultural de plantio. In: PARANHOS, S.B., cood. Cana-de-açtcar cultivo e utilizaçüo. Campinas, Fundação Cargill, 1987. v.1, p.284-332.

DOURADO NETO, D. Variabilidade espacial das alturas de chuva e irrigactio e de potenciais da soluçio do solo. Piracicaba, 1989, 180p. Dissertação (Mestrado) -Escola Superior de Agricultura "Luiz de Queiroz", Universidade de Sāo Paulo.

HAAG, H.P.; DECHEN, A.R.; CARMELLO, Q.A.C. Nutriçâo mineral da cana-de-açúcar. In: PARANHOS, S.B. coord. Cena-de-gyicar cultivo e utilizaça. Campinas, FUNDAÇÃO CARGILL, 1987. v.1, p:88-162.

HUMBERT, R.P. Planting of sugar cane. In: HUMBERT, R.P. The growing of sugar cane. Amsterdan, Elsevier, 1968. p.103-130.

JENSEN, E.S.; ANDERSEN, A.J.; THONSEN, J.D. The influence of seed-borne $\mathrm{N}$ in ${ }^{15} \mathrm{~N}$ isotope dilution studies with legmes Acta Agriculture Scandinavia, Stockholm, v.35, p.438-443, 1985.

LIMA, E.; BODDEY, R.M.; DÖBEREINER, J. Quantification of biological nitrogen fixation associated with augar cane using a ${ }^{\text {is }} \mathrm{N}$ aided nitrogen balamesoil Biology and Biochemistry, New York, v.19, p.165-170, 1987.

REIS, V.M.; OLIVARES, F.L.; DÖBEREINER, J. Improved methodology for isolation of Acetobacter diazotrophicus and confirmation of its endophytic habitat. World Journal of Microbiology and Biotecnology, v.10, p.401-405, 1994
SAMPAIO, E.V.S.B.; SALCEDO, I.H.; VICTORIA, R.L.; TRIVELIN, P.C.O. Redistribution of the nitrogen reserves of ${ }^{15} \mathrm{~N}$-enriched stem cuttings and dinitrogen fixed by 90 day-old sugarcane plants. Plant and Soil, The Hague, v.108, n.2, p.275-279, 1988.

SILVA, P.R.F.; STUTTE, C.A. Nitrogen loss in conjunction with transpiration from rice leaves as influenced by growth stage, leaf position, and $\mathrm{N}$ supply. Agronomy Journal, Madison, v.73, n.1, p.38-42, 1981.

SIMŌES NETO, D.S.; MARCOS, Z.Z. Influência da quantidade e localizaçăo da reserva nutricional do tolete sobre o desenvolvimento inicial da cana-de-açúcar. In: CONGRESSO NACIONAL DA SOCIEDADE DOS TECNICOS AÇUCAREIROS E ALCOOLEIROS DO BRASIL, 4., e CONVENÇĀO DA ACTALAC, 7. Olinda, 1987. Angis. STAB, Olinda, 1987. p.342-351.

STOLF, R.; BARBOSA, V. Quantidade de mudas nos sulcos de plantio da cana-de-açúcar em espaçamentos convencionais e estreitos: I. Qual o valor? STAB-Acícar, Alcool e Subprodutos, Piracicaba, v.9, n.1/2, p.28-30, 1990.

STOLF, R.; BARBOSA, V. Quantidade de mudas nos sulcos de plantio da cana-de-açúcar em espaçamentos convencionais e estreitos: II. Fórmulas de previsão e controle. STAB-Açúcar, Álcool e Subprodutos, Piracicaba, v.9, n.4/5, p.11-15, 1990.

STUTTE, C.A.; WEILAND, R.T.; BLEM, A.R. Gaseous nitrogen loss from soybean foliage. Agronomy Journal, Madison, v.71, n.1, p.95-97, 1979.

TAKAHASHI, D. T. Six years studies on nitrogen utilization by sugar cane plant using ${ }^{15} \mathrm{~N}$ as a tracer. In: INTERNATIONAL SOCIETY OF SUGAR CANE TECHNOLOGISTS CONGRESS, 10., Honolulu, 1959. Proceedings. Amsterdam, Elsevier Publishing Co., 1960. p.377-389.

TRIVEln, P.C.O.; SALATI, E.; MATSUI, E. Preparo de amostras para andisede ${ }^{15} \mathrm{~N}$ por espectrometria de massa. Piracicaba, CENA, 1973, 41p. (Boletim Técnico, 002).

TRIVELIN, P.C.O.; COLETI, J.T.; LARA CABEZAS, W.A.R. Efeito residual na soqueira de cana-de-açúcar do nitrogênio da uréia aplicada por via foliar na cana planta. In: SEMINÁRIO REGIONAL SOBRE TECNICAS NUCLEARES NA PRODUÇÃO DE PLANTAS AGRfCOLAS, Piracicaba, 1984. Anais. Piracicaba, CENA, 1984, p.119-124.

URQULAGA, S.; CRUZ, K.H.S.; BODDEY, R.M. Contribution of nitrogen fixation to sugar cane: Nitrogen-15 and nitrogen-balance estimates. Soil Science Society of America Journal, Madison, v.56, n.1, p.105-114, 1992. 
URQUTAGA, S.; DŌBERENER, J. Fijación biológica de nitrogeno asociada con gramineas forrajeras, cereales y cafin de azucar. In: OLIVARES, J. \& BAREA J.M. cood. Fijocion y movilizecion biologica de mutrientes. Fifeción de N y micorrizes (Vol. II). Madri, CSIC, cap.13, 1991 p.71-89.
ZAMBELLO JR., E.; AZEREDO, D.F. Adubaçăo na regifo centro-sul. In: ORLANDO FILHO, J. Nutriçâo e adubaçx̃o da cana-de-açucar no Brasil. Piracicaba, IAA Planalsucar, 1983. p.289-313.

'Recebido para publicação em 13.08.94

${ }^{2}$ Aceito para publicação em 09.01 .95 